\title{
Cystic artery pseudoaneurysm with haemobilia after laparoscopic cholecystectomy
}

\author{
K To, BA (Hons), Eric CH Lai *, MB, ChB, MRCSEd, FRACS, Daniel TM Chung, MB, ChB, MRCSEd, FRCS, \\ Oliver CY Chan, MB, ChB, MRCSEd, FRCS, CN Tang, MB, BS, FRCS
}

Department of Surgery, Pamela Youde Nethersole Eastern Hospital, Chai Wan, Hong Kong

Hong Kong Med J 2018;24:203-5

*Corresponding author: elaichun@gmail.com

DOI: $10.12809 / \mathrm{hkmj} 176236$

\section{Case presentation}

A 56-year-old man underwent laparoscopic cholecystectomy for acute cholecystitis at another hospital in December 2013. The cholecystectomy was uneventful and the patient was discharged home 3 days later. However, after hospital discharge, the patient presented with recurring upper abdominal pain, tarry stool, and fever. He was admitted to another hospital 4 weeks after the cholecystectomy because of fever, right upper quadrant pain, and haematemesis. Emergency oesophagogastroduodenoscopy and colonoscopy were performed. No bleeding source was identified. Computed tomography (CT) revealed subhepatic fluid collection; old-blood-stained fluid was drained by image-guided catheter drainage. The patient was transferred to the Department of Surgery, Pamela Youde Nethersole Eastern Hospital, Hong Kong, for further treatment.

When the patient arrived at hospital, his blood pressure was approximately $90 / 60 \mathrm{~mm} \mathrm{Hg}$ and his pulse rate was 110 beats per minute. Laboratory studies revealed the following values: haemoglobin level, $72 \mathrm{~g} / \mathrm{L}$; white blood cell count, $20.3 \times 10^{9} / \mathrm{L}$; platelet count, $388 \times 10^{9} / \mathrm{L}$; total bilirubin, 164 $\mu \mathrm{mol} / \mathrm{L}$; alanine aminotransferase, $187 \mathrm{IU} / \mathrm{L}$; and alkaline phosphatase, $337 \mathrm{IU} / \mathrm{L}$. The patient was treated with intravenous fluid hydration and was transfused with three units of packed red blood cells. He was also given a course of antibiotics. Abdominal CT showed a cystic artery pseudoaneurysm of $1.22 \times 1.96 \times 1.38 \mathrm{~cm}$ (anterior-posterior $\times$ transverse $\times$ longitudinal dimensions). Two subhepatic collections with haematoma were also visible, over the gallbladder fossa and below hepatic segment 6 . Selective right hepatic artery angiography revealed a pseudoaneurysm at the cystic artery. This aneurysm was embolised with stainless steel coils (Fig 1). The catheter for subhepatic collection drainage was then replaced with one with better positioning. Endoscopic retrograde cholangiopancreatography was performed the next day. The cholangiogram showed a dilated biliary tree with haemobilia; most of the blood clots were extracted using a balloon. A cystic duct stump leak was observed after blood clot removal, and a 10-cm-long 11.5-F biliary stent was inserted for biliary drainage (Fig 2). Liver function improved gradually. The patient was discharged
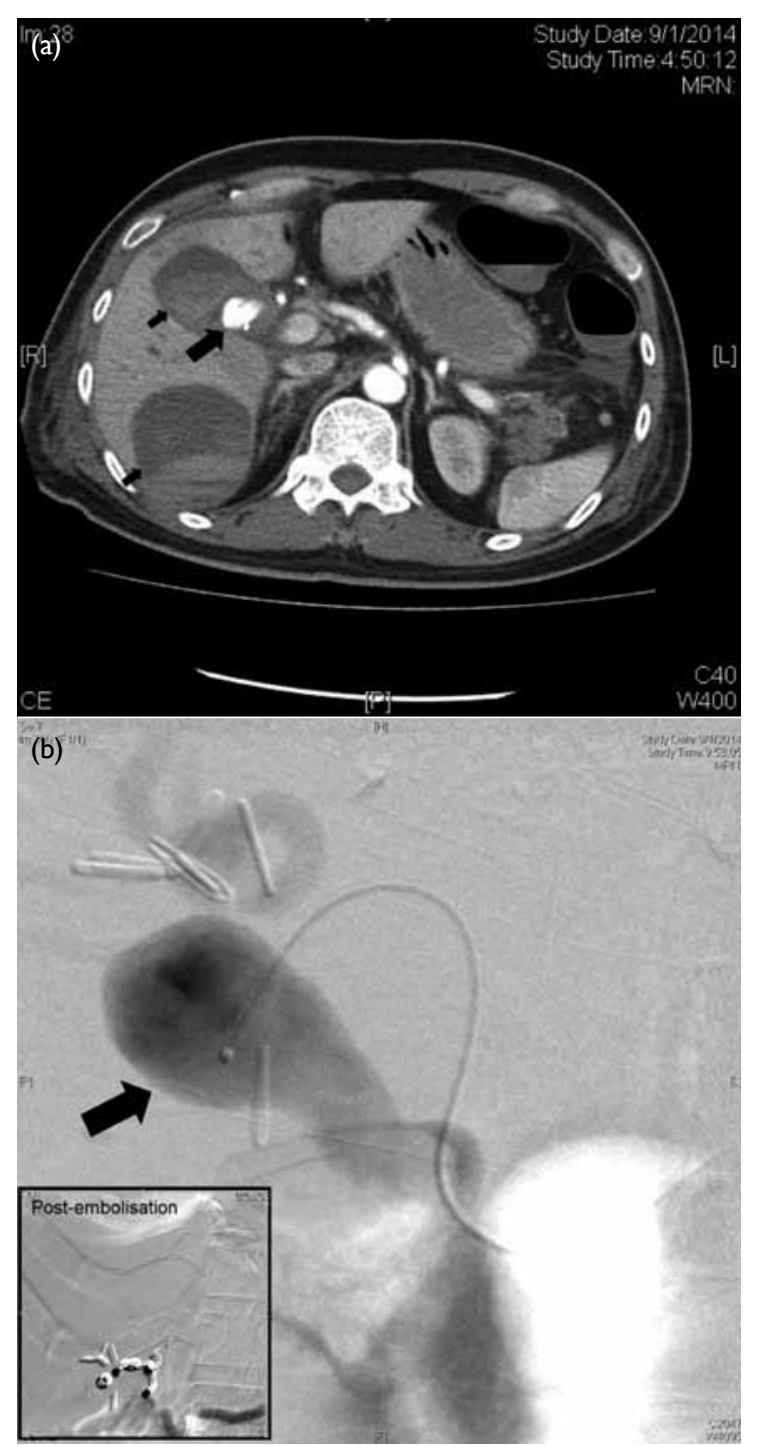

FIG I. (a) Computed tomogram showing pseudoaneurysm of the cystic artery (large arrow), and two subhepatic collections with haematoma over the gallbladder fossa and below hepatic segment 6 (small arrows). (b) Angiogram showing pseudoaneurysm of the cystic artery (arrow) and the pseudoaneurysm after embolisation (inset) 


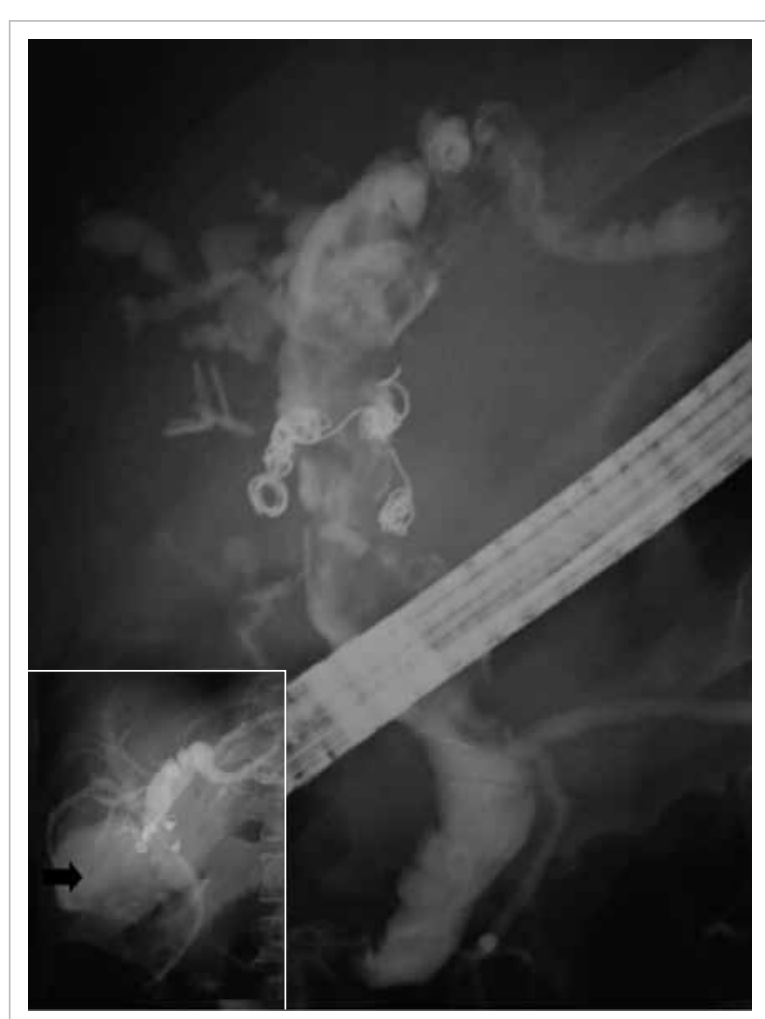

FIG 2. Cholangiogram showing haemobilia before blood clot removal, and cholangiogram (inset) showing cystic duct stump leakage (arrow) after blood clot removal and controlled with biliary stenting

from hospital 2 weeks after admission.

Follow-up CT no longer showed pseudoaneurysm and instead showed a resolving collection. Endoscopic retrograde cholangiopancreatography with stent removal was performed 3 months later. The cholangiogram showed a normal biliary tree. The patient recovered and liver function test results were normal.

\section{Discussion}

Hepatic artery or cystic artery pseudoaneurysms are rare complications of laparoscopic cholecystectomy, with cystic artery involvement being reported much less frequently in the literature. Pseudoaneurysm formation is a consequence of vascular injury; important causes include arterial access procedures, accident trauma, and surgical trauma. ${ }^{1}$ Two-thirds of cases are iatrogenic. ${ }^{1}$ With the advent of laparoscopic cholecystectomy, iatrogenic hepatobiliary injury is now another cause. Concomitant formation of cystic artery pseudoaneurysm and cystic duct stump leak is a rare complication of laparoscopic cholecystectomy. The majority of pseudoaneurysms present within 6 weeks after the operation., ${ }^{2,3}$

We have reported a case of laparoscopic cholecystectomy that was complicated by a cystic artery pseudo-aneurysm and a cystic duct stump bile leak, which were managed with angiographic coil embolisation and endoscopic biliary drainage, respectively. The patient presented with the classic Quincke's triad of haemobilia, namely upper gastrointestinal bleeding, right upper quadrant pain, and obstructive jaundice. The aetiology most likely originated from the infected fluid collection after cholecystectomy, which caused a series of events, including cystic duct stump leak, cystic artery pseudoaneurysm, and haemobilia, in that order. First, bile leakage is a potential complication of cholecystectomy and the cystic duct stump is the most common site of leakage. ${ }^{4}$ The contributing factor of cystic duct stump leak in the current case was likely cystic duct stump necrosis secondary to mechanical or thermal injury during cholecystectomy, as well as adjacent infection. Second, haemobilia can occur secondary to a cystic artery pseudoaneurysm, although extremely rarely. Artery pseudoaneurysm is a continuous inflammatory process that leads to erosion in the elastic and muscular components of the arterial wall, ultimately resulting in pseudoaneurysm formation. The likely precipitating factors in the current case include initial clip encroachment of the vasculature, mechanical or thermal injury, and continuous inflammation due to the adjacent infected bile or collection.

Pseudoaneurysm can present with bleeding in the form of haemobilia, haematemesis, or melaena. In the current case, upper gastrointestinal bleeding from haemobilia resulted from the cystic artery pseudoaneurysm's communication with the cystic duct. The resulting symptoms were typical of Quincke's triad of upper abdominal pain, upper gastrointestinal haemorrhage, and jaundice. ${ }^{5}$ However, these symptoms are present collectively only in a minority (32\%-40\%) of patients. ${ }^{5}$ Thus, detection relies heavily on both clinical reasoning and imaging techniques. If intraabdominal collection or haemorrhage is suspected clinically, arterial-phase $\mathrm{CT}$ is appropriate to detect any pseudoaneurysm. Since gastrointestinal haemorrhage is one of the presentations, urgent oesophagogastroduodenoscopy may be arranged first to rule out any suspected upper gastrointestinal pathology. However, as in the current case, if that procedure fails to show any bleeding source, urgent CT should be considered. Close observation and timely arrangement of appropriate procedures are essential.

Prompt recognition with adequate management was very important in the current case. The treatment of our patient included five objectives: achieving haemostasis, controlling the cystic duct stump leak, relieving obstructive jaundice, controlling the infection with antibiotics, and draining the intra-abdominal collection. 
Untreated haemobilia poses an immediate threat to life. It can lead to acute haemodynamic instability, necessitating detection, access, and control of the pseudoaneurysm. Arterial-phase CT is a good initial non-invasive mode of detection of laparoscopic cholecystectomy complications. It can be used to evaluate intra-abdominal collection, biliary tree dilatation, and possible bile duct injury, and to visualise pseudoaneurysms or haemorrhage. Arterial-phase CT also allows a three-dimensional assessment of the bile duct and vasculature. Selective arterial angiography can provide a real-time evaluation of pseudoaneurysms and bleeding. At the same time, it can provide the chance of immediate therapeutic intervention. Transarterial embolisation is the treatment of choice for haemostasis, and a high success rate, of $75 \%$ to $100 \%$, has been reported. ${ }^{2,3,5}$ When bleeding control by embolisation fails, repeated sessions of transarterial embolisation for haemostasis or surgical intervention to repair or ligate the artery is necessary. Endoscopic retrograde cholangiopancreatography with stent placement or sphincterotomy is highly effective in diagnosing haemobilia, controlling cystic duct stump leakage, and relieving obstructive jaundice. We favoured stenting over sphincterotomy because of a presumed lower risk of immediate complications.

The lessons to be learnt from this case include the importance of (1) meticulous surgical techniques (such as good haemostasis, careful use of powered devices, proper use of endoclips, and adequate drainage of the operative field), and (2) early recognition and prompt management.

In conclusion, cystic artery pseudoaneurysm is a rare, potentially life-threatening complication of laparoscopic cholecystectomy, and prompt recognition and treatment are essential. Haemobilia may be present many weeks after the initial injury.

\section{Declaration}

The authors have no conflicts of interest to disclose.

\section{References}

1. Green MH, Duell RM, Johnson CD, et al. Haemobilia. Br J Surg 2001;88:773-86.

2. Senthilkumar MP, Battula N, Perera M, et al. Management of a pseudo-aneurysm in the hepatic artery after a laparoscopic cholecystectomy. Ann R Coll Surg Engl 2016;98:456-60.

3. Nicholson T, Travis S, Ettles D, et al. Hepatic artery angiography and embolization for hemobilia following laparoscopic cholecystectomy. Cardiovasc Intervent Radiol 1999;22:20-4.

4. Lau WY, Lai EC, Lau SH. Management of bile duct injury after laparoscopic cholecystectomy: a review. ANZ J Surg 2010;80:75-81.

5. Merrell SW, Schneider PD. Hemobilia-evolution of current diagnosis and treatment. West J Med 1991;155:6215 . 